\title{
Pediatric Lumbar Epidural Abscess Combined with Cauda Equina Syndrome: Case Report
}

\author{
Moon-Chan Kim, Hung-Tae Jung \\ Department of Orthopaedic Surgery, Bumin Hospital, Busan, Korea
}

Pyogenic epidural abscess is a very rare disease. Once it occurs, it promptly progresses and can cause neurologic paralysis. Mean age of onset has been reported to be 57 years. Here we report making a diagnosis of pyogenic lumbar epidural abscess accompanying cauda equina syndrome in a 10-year-old girl. We treated this case successfully with surgical drainage and antibiotics. We report our case with a review of the literature.

Key Words: Lumbar epidural abscess, Couda equina syndrome

\section{Introduction}

Pyogenic epidural abscess is a very rare disease without unique symptoms. Once it occurs, however, it promptly progresses and can also cause neurologic paralysis. In some cases, delayed diagnosis may lead to the occurrence of catastrophic complications. An early diagnosis and immediate treatment is thus mandatory [1-3]. The incidence has been reported to be 0.2 to 1.2 per 10,000 hospitalized patients per year, and has recently been increasing [1]. The reasons for the increase might include the increased number of older individuals in an aging population, the increasing use of spinal instrumentation, overuse of antibiotics, and the increased number of immunocompromised individuals in the population such as patients with diabetes mellitus or cancer. Most cases occur in adults at an average age of 57 years (range, 2 to 81 years). Although the disease is rare, mortality and morbidity have been reported to be higher in pediatric cases than in adult ones [1]. We made a diagnosis of a case of pyogenic lumbar epidural abscess accompanying cauda equina syndrome in a 10 -year-old girl. We suc- cessfully treated her with surgical drainage and antibiotics. Here, we report our case with a review of the literature.

\section{Case Report}

A 10-year-old girl received conservative treatment for a 10-day-history of high fever, low back pain and radiating pain in both legs at a local clinic prior to her outpatient visit. Due to a lack of improvement in symptoms, however, the patient was transferred to the emergency room of our medical institution. At the time of her outpatient visit, there was saddle anesthesia in areas adjacent to the perineal and anal regions. The patient also presented with findings suggestive of bladder and bowel dysfunction. In her past medical history, the patient had no notable findings or underlying diseases. At the time of her outpatient visit, she presented with blood pressure of $110 / 80 \mathrm{~mm} \mathrm{Hg}$, pulse rate of 100 times/min, and a body temperature of $38.2^{\circ} \mathrm{C}$. On hematologic examination, findings were a white blood cell count of $16,200 / \mu \mathrm{l}$ (seg $68 \%$ ), an erythrocyte sedimentation rate (ESR) of $52 \mathrm{~mm} / \mathrm{hr}$ (normal, 0 to $20 \mathrm{~mm} / \mathrm{hr}$ ) and an hs-Creactive protein (CRP) of $14.65 \mathrm{mg} / \mathrm{dl}$ (normal, 0 to 0.5

Received Feb 1, 2010; 1st Revised Mar 17, 2010; 2nd Revised Mar 23, 2010; 3rd Revised Apr 11, 2010; Accepted Apr 11, 2010

Corresponding author: Moon-Chan Kim, MD

Department of Orthopaedic Surgery, Boomin Hospital, 380-4 Deokcheon-1 dong, Buk-gu, Busan 616-819, Korea

Tel: +82-51-330-3000, Fax: +82-51-330-3242, E-mail: moon_chan@hanmail.net 
$\mathrm{mg} / \mathrm{dl}$ ). On magnetic resonance imaging (MRI) scans that were taken at the time of admission, there were lesions at spinal levels L3 to L5 vertebra which were suspected to be an epidural abscess (Fig. 1). Then, during surgery for a mass effect of the thecal sac, hemilaminotomy and drainage were done under microscopy. Surgical findings included a yellow-colored, abscess-like fluid under the yellow ligament. On culture testing, causative bacteria were identified as Staphylococcus aureus (Fig. 2). Immediately after surgery, all symptoms disappeared. There were no neurological deficits. For the ensuing four postoperative weeks, IV antibiotics (including 1st-generation ceftezole) were used. During a period ranging from postoperative weeks 4 to 6 weeks, oral antibiotics (1st-generation cefaclor) were used. In postoperaitve week 6, white blood cell (WBC) counts, ESR and CRP were all found to be normal. A 2nd lumbar MRI was done, and disappearance of the preoperative abscess was confirmed. During a 6-month follow-up period, there was no recurrence. The patient has been able to maintain a normal life (Fig. 3).

\section{Discussion}

Pyogenic epidural abscess is a very rare disease. In some cases, a delayed diagnosis may lead to the occurrence of catastrophic complications. Mortality and morbidity are much higher in pediatric patients than in adults. According to Baker et al. [1], the incidence has been reported to be 0.2 to 1.2 per 10,000 hospitalized patients, and has recently been increasing. Among causative bacteria, Staphylococcus aureus is the most common. This is followed by Streptococcus, S. epidermis and Escherichia coli [2]. The pathophysiology can be classified into direct inoculation and hematogeneous spread. In our case, the causative bacteria were found to be Staphylococcus aureus. Symptoms manifested ten days before as acute disease, based on which a hematogenous infection might be the etiology. This disease occurs most commonly in the thoracic region, followed by the lumbar, cervical and lumbosacral regions. In approximately $80 \%$ of cases, it also occurs in the posterior epidural space and less so in the lateral epidural space. Disease in the
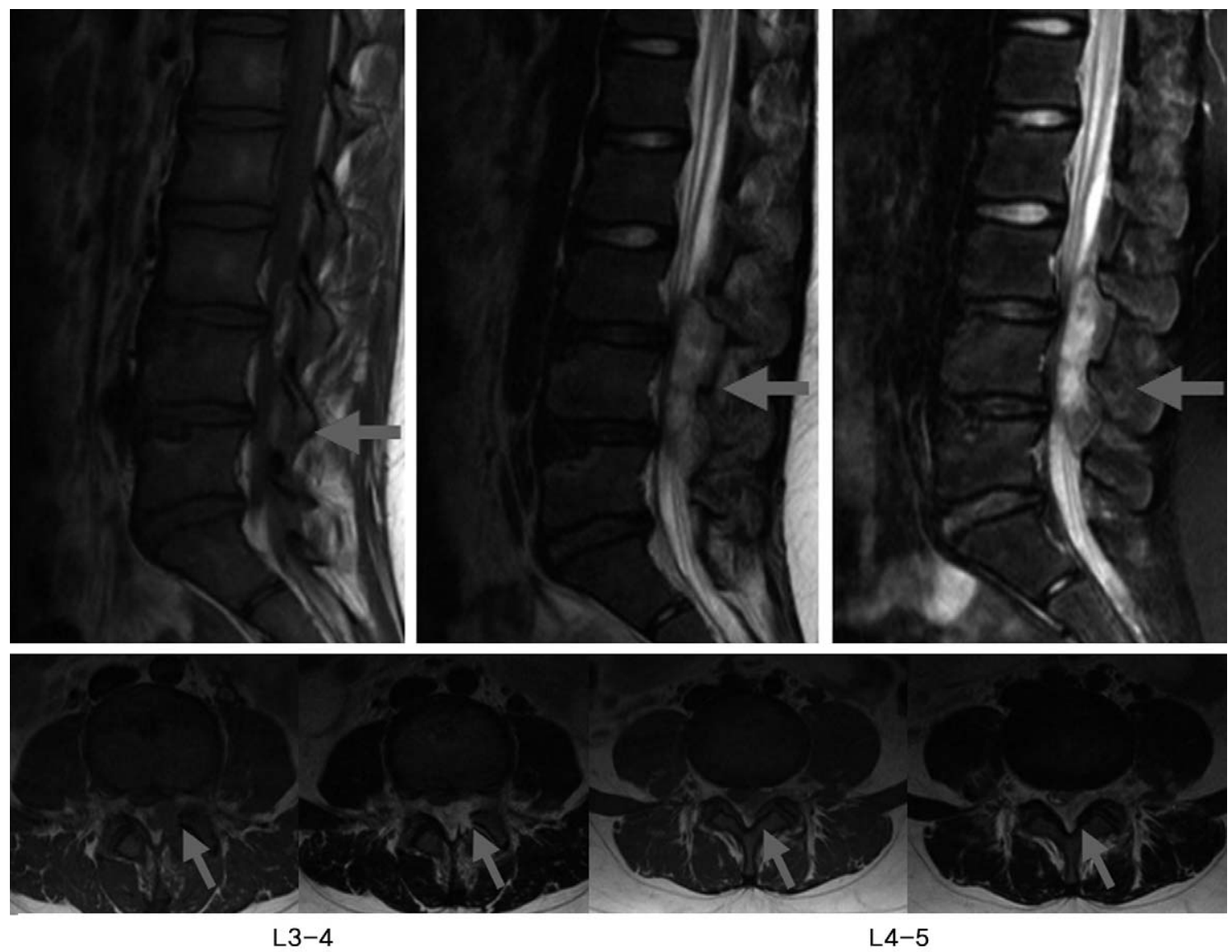

Fig. 1. Magenetic resonance image showing a posterior epidural mass, which was displacing and compressing the cauda equina at L3-4 to L4-5 levels. 


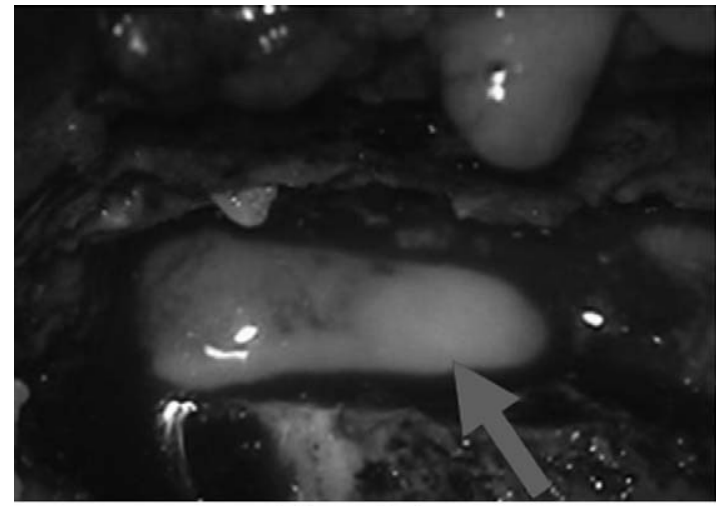

Before drainage

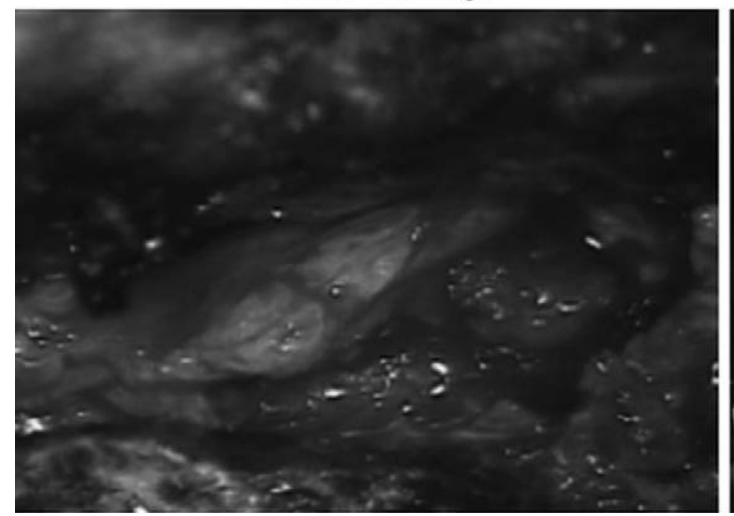

Fig. 2. Intraoperative microscopic finding documenting thick-purulent yellowish pus collection in the epidural spaces. A hemilaminotomy was done and the epidural abscess drained.

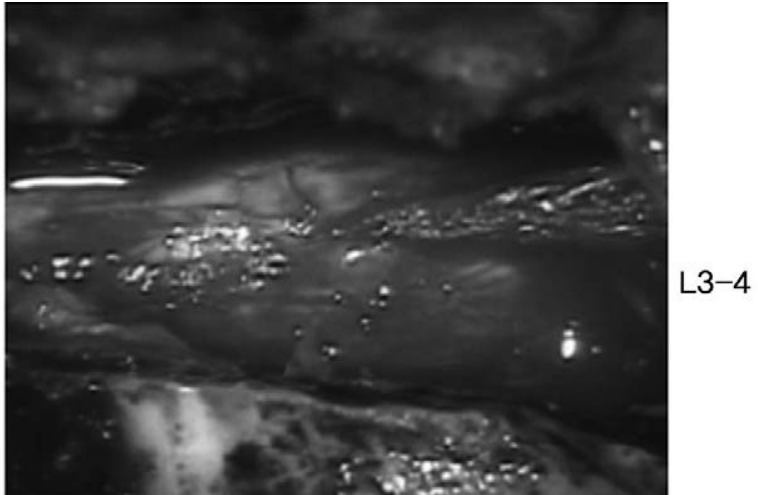

After drainage

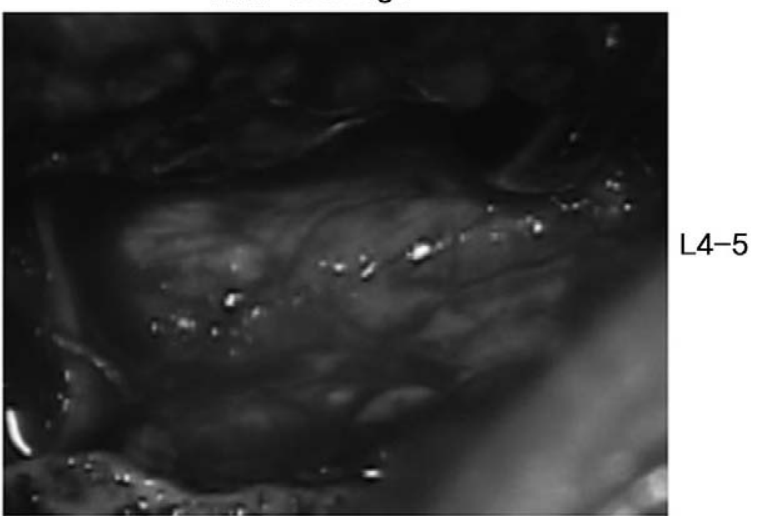

L4-5 anterior epidural space has been reported to occur in approximately $10 \%$ of cases [3]. The disease commonly occurs in the epidural space, which is a continuous structure without a defensive barrier. The disease occurs more commonly in multiple segments consisting of 3 to 5 spinal segments rather than in a single segment [1]. In our case, the epidural abscess compressed the spinal cord between the posterior regions of L3 and L5. Based on the clinical symptoms of epidural abscess, an early diagnosis is very difficult. In most cases, there are such symptoms as lower back pain, radiating pain and progressive neurologic deficit. But there is a variability in the progression pattern and its duration. There are also some patients who do not complain of fever or lower back pain and those who have chronic progression [2,3]. In our case, the patient presented with a persistent progression of lower back pain and high fever ten days prior to her outpatient visit. At the time of the outpatient visit, there was a saddle anesthesia in the areas adjacent to the perineal and anal regions. On physical examination, the patient presented with lower back tenderness. A straight leg raising test showed a $60^{\circ}$ range of motion on the right side and a $40^{\circ}$ range on the left side. A hematolog- ic examination commonly shows the increased WBC counts, ESR and the increased level of CRP. It is also known that etiological pathogens can be identified from a blood culture in approximately $60 \%$ of cases [3]. In our case, however, there were negative results to a blood culture test. Also, causative bacteria were identified on a bacterial culture test following drainage. According to Bertino et al. [4], bone scan, myelogram and MRI scans are useful in making a diagnosis of intraspinal abscess and vertebral osteomyelitis. Also, Lang et al. [5] reported that contrastenhanced findings are typically seen in a gadolinium-DTPA MRI scan. Also, in our case, the diagnosis was made at earlier times following an MRI. Under the suspicion of epidural abscess, an emergency operation was performed. Treatments can be classified into medical and surgical. Excluding cases in which the abscess was extensively present in a widespread area of the spinal canal, there are those in which there are no severe neurologic deteriorations, those in which a complete paralysis is persistently present for more than three days, and those in which surgical treatment was refused. Concomitant use of two treatment modalities is recommended [6-9]. Surgical modalities are determined 

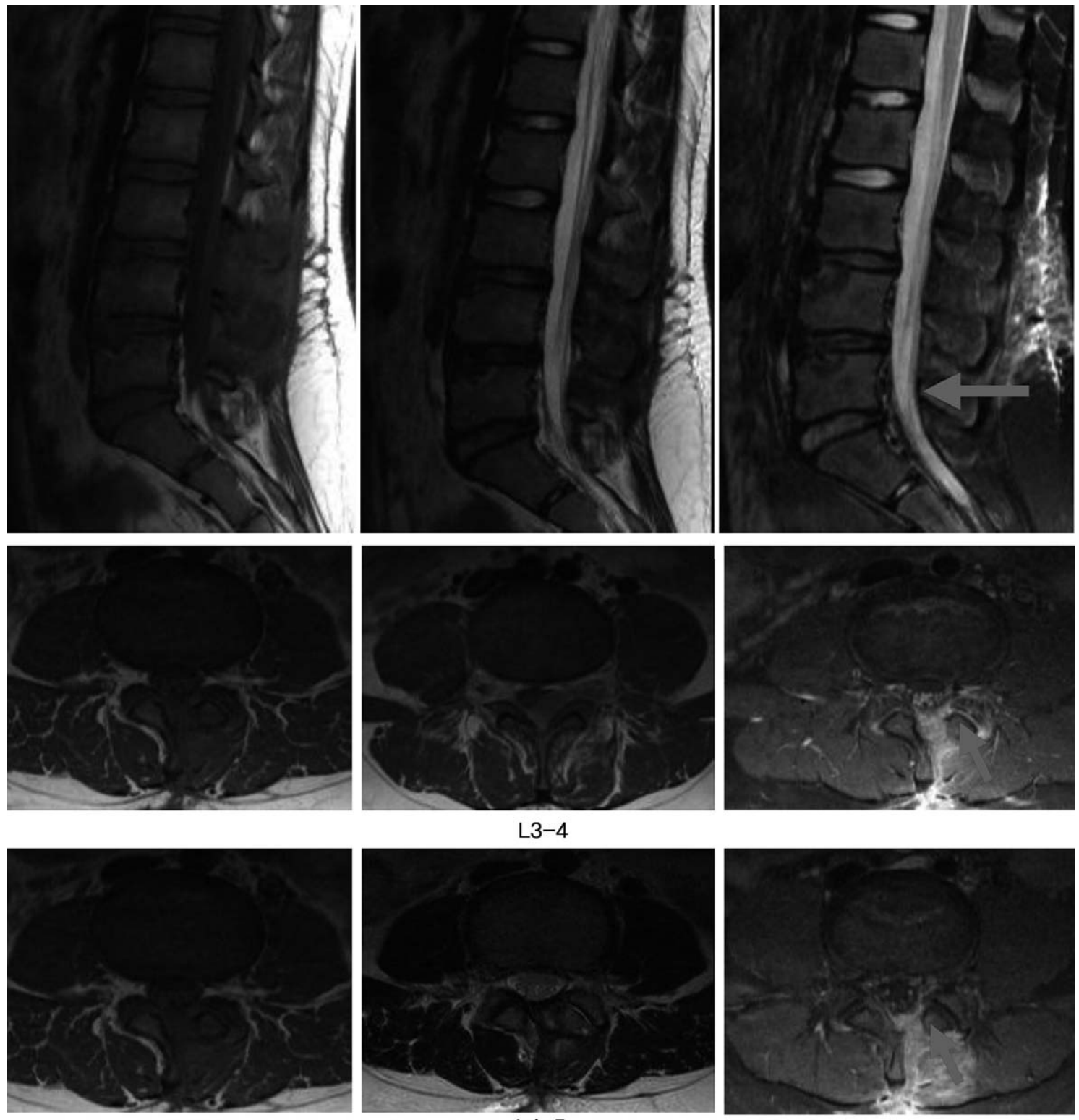

L4-5

Fig. 3. Postoperative contrast magenetic resonance images after 6 mo demonstrating complete removal and no recurrence.

based on the location. But most cases are located posterior to the spinal cord. Drainage via a posterior approach is universally done. In cases in which vertebral osteomyelitis is concurrently present, anterior and posterior decompression should concomitantly be used. Spinal fusion has also been found to be mandatory for cases in which spinal instability occurred [10]. Following surgery, antibiotics should be used for at least eight weeks in cases in which vertebral osteomyelitis was concurrently present and for at least four weeks in other cases [10]. In our case, the posterior lamino- tomy and drainage were done for the epidural abscess. Due to a lack of concurrent presence of vertebral osteomyelitis, oral antibiotics were used until postoperative 4 week. During postoperative 4-6 weeks, oral antibiotics were used. On postoperative 6 week, a 2nd lumbar MRI was done, and disappearance of the preoperative abscess was confirmed. We made a final diagnosis of pyogenic lumbar epidural abscess accompanying cauda equina syndrome in a 10-year-old girl. We successfully treated this case with surgical drainage and antibiotics. 


\section{REFERENCES}

1. Baker AS, Ojemann RG, Swartz MN, Richardson EP Jr. Spinal epidural abscess. N Engl J Med 1975;293:463-8.

2. Heusner AP. Nontuberculous spinal epidural infections. $\mathrm{N}$ Engl J Med 1948;239:845-54.

3. Rigamonti D, Liem L, Sampath P, et al. Spinal epidural abscess: contemporary trends in etiology, evaluation, and management. Surg Neurol 1999;52:189-96.

4. Bertino RE, Porter BA, Stimac GK, Tepper SJ. Imaging spinal osteomyelitis and epidural abscess with short TI inversion recovery (STIR). AJNR Am J Neuroradiol 1988; 9:563-4.

5. Lang IM, Hughes DG, Jenkins JP, St Clair Forbes W, McKenna F. MR imaging appearances of cervical epidural abscess. Clin Radiol 1995;50:466-71.
6. Mampalam TJ, Rosegay H, Andrews BT, Rosenblum ML, Pitts LH. Nonoperative treatment of spinal epidural infections. J Neurosurg 1989;71:208-10.

7. Davis DP, Wold RM, Patel RJ, et al. The clinical presentation and impact of diagnostic delays on emergency department patients with spinal epidural abscess. J Emerg Med 2004;26:285-91.

8. Bluman EM, Palumbo MA, Lucas PR. Spinal epidural abscess in adults. J Am Acad Orthop Surg 2004;12:155-63.

9. Sampath P, Rigamonti D. Spinal epidural abscess: a review of epidemiology, diagnosis, and treatment. J Spinal Disord 1999;12:89-93.

10. Nussbaum ES, Rigamonti D, Standiford H, Numaguchi Y, Wolf AL, Robinson WL. Spinal epidural abscess: a report of 40 cases and review. Surg Neurol 1992;38:225-31. 